\title{
Influence of commensal microbiota on aggressive behaviors
}

Natsuru Watanabe, Katsunaka Mikami, Keitaro Kimoto, Fumiaki Akama, Yuji Aiba, Kenji Yamamoto, Hideo Matsumoto

Department of Psychiatry, Tokai University School of Medicine, Kanagawa, Japan

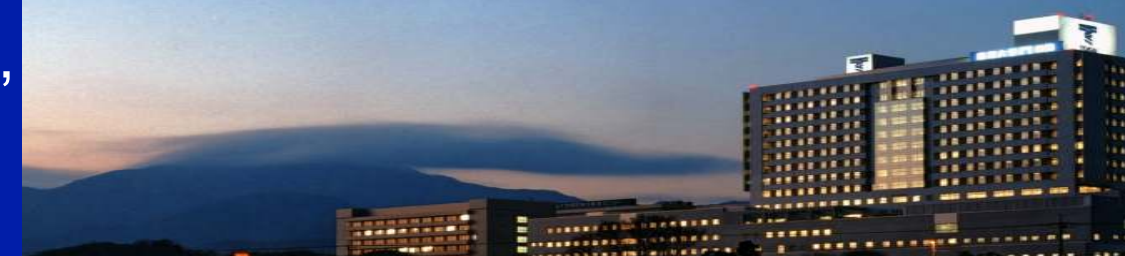

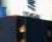
10

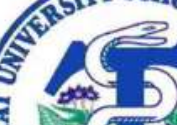

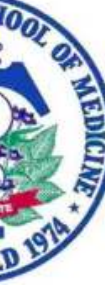

Introduction

Increasing evidences suggest influence of microbiota-gut-brain axis on mental status and behavior. In fact, our previous study on germfree (GF) mice and commensal fecal microbiota-associated mice (EX-GF) demonstrated that commensal microbiota decreased both spontaneous locomotor activity and anxiety-like behavior of the host. These findings provided influence of commensal microbiota on the host behaviors and mental state.

This study aimed to examine the influence of commensal microbiota on the host aggressive behavior which is a characteristic of neurodevelopmental disorders.

\section{Methods}

Social interaction test was used to analyze aggressive behaviors in gnotobiotic BALB/c mice in a sterile isolator. This interaction test was performed in the Open-filed box, and GF mice and same group of castrated GF mice were setting opposite side. Their behaviors were recorded by a video for 10 minutes. Behavioral test of the mice were performed at 8 and 10 weeks of age. Aggressive behaviors evaluated tail rattling, attacks, chasing, wrestling and aggressive grooming (Table 1). In the same way, aggressive behaviors of EX-GF and same group of castrated EX-GF were observed.

The monoamine levels in several regions of the brain were measured in GF and EX-GF mice immediately after interaction test.

IBM SPSS Statistics version 20 for Windows was used for data analysis.

Table 1 Definition of variables aggressive behaviors in mice Tail rattling : Rapid lateral quivering or thrashing of the tail Attacks : Biting of the opponent mouse

Aggressive grooming : Vigorous grooming of the opponent mouse from lateral position, using teeth and forepaws

Wrestling : Vigorous shoving and sparring when both animals take on an upright posture

Chasing : A rapid pursuit of the standard opponent by the test male, with or without physical contact
The frequency of mice with aggressive behaviors was significantly higher in the group of GF mice than the group of EX-GF mice (Table 2). Turnover rates of serotonin metabolite 5-hydroxyindoleacetic acid/ serotonin (5-HIAA/5HT) had high tendency in the brain of the EX-GF mice than in that of the GF mice（Table 3).

Table 2 Comparison of aggression behavioral characteristics in GF mice and EX-GF mice

$\begin{array}{cc}\text { GF mice } & \text { EX-GF mice } \\ (n=30) & (n=30)\end{array}$

\begin{tabular}{lcc}
\hline Tail rattling, $n$ & 13 & 0 \\
Attacks, $n$ & 10 & 0 \\
Aggressive grooming, $n$ & 11 & 0 \\
Wrestling, $n$ & 0 & 0 \\
Chasing, $n$ & 7 & 0 \\
Observed aggression, & $15(50)$ & $0(0)$ \\
$n(\%)$ & &
\end{tabular}

Categorical variables were compared using Fisher's exact test.

Table 3 5-HIAA/5HT turnover rates in various regions of the brain

\begin{tabular}{lccc}
\hline & $\begin{array}{c}\text { GF mice } \\
(n=9)\end{array}$ & $\begin{array}{c}\text { EX-GF mice } \\
(n=9)\end{array}$ & $P$ \\
\hline Prefrontal cortex & $0.07(0.02)$ & $3.54(0.02)$ & $<0.001$ \\
Hippocampus & $0.89(0.03)$ & $1.62(0.08)$ & $<0.001$ \\
Striatum & $0.85(0.03)$ & $8.37(0.38)$ & $<0.001$ \\
Brainstem & $1.04(0.04)$ & $1.62(0.09)$ & $<0.001$ \\
\hline
\end{tabular}

All data represent the means \pm standard error (SE). Continuous variables were compared using the Mann-Whitney $U$ test.

\section{Conclusion}

These results strongly support the influence of the gut microbiota on the host aggressive behaviors. Our studies help to develop the complex interactions and pathways of microbiota-gut-brain axis on mental status and behavior.

\section{Disclosures}

This study has no fund. The authors have no conflicts of interest directly relevant to the content of this article. 